\title{
Prostaglandin Reductase 2 Modulates ROS- Mediated Cell Death and Tumor Transformation of Gastric Cancer Cells and Is Associated with Higher Mortality in Gastric Cancer Patients
}

\author{
Emily Yun-Chia Chang, ${ }^{*}$ Shu-Huei Tsai, ${ }^{\dagger}$ \\ Chia-Tung Shun, ${ }^{\ddagger}$ Siow-Wey Hee, ${ }^{\dagger}$ \\ Yi-Cheng Chang, ${ }^{\dagger}$ Yun-Chih Tsai, $^{\dagger}$ \\ Jaw-Shiun Tsai, § Hsian-Ju Chen," Jia-Wei Chou," \\ Shih-Yao Lin, " and Lee-Ming Chuang ${ }^{\dagger \|}$ \\ From the Institute of Molecular Medicine $e^{*}$ and the Department of \\ Medicine, College of Medicine, National Taiwan University, \\ Taipei; the Departments of Internal Medicine, ${ }^{\dagger}$ Forensic Medicine \\ and Pathology, ${ }^{\neq}$and Family Medicine, ${ }^{\S}$ National Taiwan \\ University Hospital, Taipei; and AbGenomics BV, Taiwan \\ Branch, Neibu Taipei, Taiwan
}

Various prostanoids and peroxisome proliferator-activated receptor $\gamma(\operatorname{PPAR} \gamma)$ ligands play an important role in gastric cancer. Previously, we demonstrated that prostaglandin reductase 2 (PTGR2) catalyzes the reduction of the PPAR $\gamma$ ligand 15-keto-PGE 2 into 13,14-dihydro-15-keto-PGE ${ }_{2}$. Here, we present functional data and clinical relevance for the role of PTGR2 in gastric cancer. Using lentiviral technology in AGS and SNU-16 gastric cancer cell lines, we either down-regulated or overexpressed PTGR2. In vitro analysis showed that PTGR2 knockdown resulted in decreased proliferation rate and colony formation, and in vivo xenograft models showed slower growth of tumors. Mechanistically, PTGR2 knockdown induced cell death, altered mitochondrial function, and increased reactive oxygen species production, which led to activation of ERK1/2 and caspase 3, with increased Bcl-2 and suppressed Bax expression. PTGR2 overexpression showed the opposite outcomes. Clinically, immunopathological staining showed strong PTGR2 expression in the gastric tumor portion, relative to nearby nontumor portions, and its expression negatively correlated with survival of patients with intestinal-type gastric cancer. Finally, in contrast to PTGR2-overexpressing cells, PTGR2-knockdown cells were more sensitive to cisplatin and 5-fluorouracil. Taken together, our findings not only provide func- tional and mechanistic evidence of the involvement of PTGR2 in gastric cancer, but also provide clinical observations affirming the significance of PTGR2 in gastric cancer and suggesting that PTGR2-target based therapy is worth further evaluation. (Am J Pathol 2012, 181:13161326; bttp://dx.doi.org/10.1016/j.ajpath.2012.07.006)

Gastric cancer is one of the leading causes of cancerrelated death, especially in Asia. Given that patient prognosis remains poor, and because of chemoresistance, the need for new therapeutic targets is obvious. ${ }^{1-3}$ Various prostaglandins and prostaglandin synthases are known to play distinct roles in tumor progression and cancer metastasis. ${ }^{4,5}$ Peroxisome proliferator-activated receptor $\gamma(\operatorname{PPAR} \gamma)$ ligands, either independently or through PPAR $y$ activation, are also known to influence cancer progression. In particular, a number of reports have proposed that PPAR $\gamma$ ligands in general suppress gastric cancer cell growth and induce apoptosis. ${ }^{6-11} \mathrm{~A}$ recent genome-wide association study identified the region encoding prostaglandin E receptor 4 as a novel susceptibility locus for gastric cancer. ${ }^{12}$ Thus, enzymes regulating prostaglandin metabolism and the related PPAR $\gamma$ activity might be involved in gastric carcinogenesis.

Prostaglandin reductase 2 (PTGR2) catalyzes the NADPHdependent reduction of 15-keto-PGE ${ }_{2}$ into the downstream metabolite 13,14-dihydro-15-keto-PGE ${ }_{2}{ }^{13,14}$ We have previously demonstrated that $15-$ keto-PGE 2 acts as a natural ligand of PPAR $\gamma$ and that overexpressing PTGR2 results in the suppression of PPAR $\gamma$-mediated adipocyte differentiation. ${ }^{13}$ Recently, 15-keto-PGE ${ }_{2}$ was proposed to trigger the translocation of the proapoptotic protein Bax to mitochondria, thereby inducing apoptosis. A stable docking model of

Supported by the National Science Council of Taiwan (grant NSC 1002314-B-002-068-MY3 to L.-M.C.)

Accepted for publication July 2, 2012.

Address reprint requests to Lee-Ming Chuang, M.D., Ph.D., Department of Internal Medicine, National Taiwan University Hospital, 7 ChungShan South Road, Taipei, Taiwan. E-mail: leeming@ntu.edu.tw. 
15-keto-PGE ${ }_{2}$ with Bax was also suggested. ${ }^{15}$ Given the link between 15 -keto-PGE ${ }_{2}$ and other prostanoids with cellular processes that dictate cell fate, we hypothesized that PTGR2 may play a role in gastric cancer and may be involved in the regulation of cancer cell death.

Apoptosis and necrosis are two different major pathways for cell death, ${ }^{16,17}$ and mitochondria are one of the most important organelles that play an essential role in the regulation of cell death processes. ${ }^{18,19}$ Extensive research has shown that mitochondria are involved in cell death processes at several levels, and that cancer cells are often adapted to avert mitochondria-mediated cell death. In addition to the regulation of $\mathrm{Bcl}-2$ family proteins, ${ }^{15,20}$ an important mitochondria-mediated mechanism of cell death is through the generation of reactive oxygen species (ROS), which can result in both apoptosis and necrosis.

Under normal conditions, ROS are generated as a consequence of aerobic metabolism; $1 \%$ to $2 \%$ of electrons usually leak from the mitochondria during the electron-transport steps of ATP production to form superoxide free radicals. When the endogenous antioxidant defense systems fails to counteract the potentially injurious actions of ROS, the cellular redox homeostasis is altered because of excess ROS; the resulting oxidative stress could trigger the JNK/ERK-dependent apoptotic cell death cascade, as well as necrosis. ${ }^{21-25}$ Cancer cells often display a distinctive adaptation, known as the Warburg effect, reducing the use of mitochondrial respiration by switching from aerobic to anaerobic glucose metabolism (glycolysis) even in the presence of normal oxygen levels. Thus, generation of ATP in cancer cells is not a mitochondria- and oxygen-dependent process, and the cells evade both ATP depletion and cell death signaling induced by excess ROS generation. ${ }^{26,27}$

In the present study, we investigated the hypothesis that PTGR2 may be involved in modulating the oncogenic characteristics of gastric cancer cells. We report not only functional and mechanistic insights into the oncogenic role of PTGR2 in modulating gastric cancer cell death and tumor transformation, but also clinical validation and potential chemotherapeutic benefits of targeting PTGR2.

\section{Materials and Methods}

\section{Cell Culture}

Human gastric carcinoma cell lines AGS and SNU-16 cells were purchased from the Bioresource Collection and Research Center (BCRC, Hsinchu, Taiwan). AGS and SNU-16 cells were cultivated in RPMI 1640 medium supplemented with 10\% fetal bovine serum (Gibco; Life TechnologiesInvitrogen, Carlsbad, CA), 2 mmol/L glutamine, and 100 units $/ \mathrm{mL}$ of penicillin and streptomycin. The cellular environment was maintained at $5 \% \mathrm{CO}_{2}$ and $37^{\circ} \mathrm{C}$ in an incubator. The lentiviral-transduced stable cell lines were cultured in growth medium containing $2 \mu \mathrm{g} / \mathrm{mL}$ puromycin (InvivoGen, San Diego, CA).

\section{Lentiviral Transduction}

The lentiviral expression vector pLKO.1shLuc was used as a cloning vector for constructing pLKO.1-shPTGR2 containing 5'-GAAAGCAGATAGTTTGCATTT-3'. pSIN$M_{C S^{28}}$ was used as the cloning vector for constructing PTGR2 overexpression plasmid pSIN-PTGR2. Virus was harvested after cotransfecting pLKO.1-Luc or pLKO.1shPTGR2, or pSIN or pSIN-PTGR2 plasmid, with pMDG and pCMV- $\Delta$ R8.91 into 293T cells using Lipofectamine 2000 transfection agent (Life Technologies-Invitrogen). At 48 hours after transfection, lentiviral supernatants were harvested and a QuickTiter lentivirus titer kit (Cell Biolabs, San Diego, CA) was used for quantifying lentiviral titer. Using multiplicity of infection $(\mathrm{MOI})=2$, the AGS and SNU-16 gastric cancer cell lines were infected with lentiviral vector particles to generate PTGR2-knockdown or PTGR2-overexpressing cell lines.

\section{Generation of Anti-PTGR2 Antibody}

Three 6- to 8-week-old male BALB/c mice were immunized with $50 \mathrm{mg}$ recombinant human PTGR2, followed by three rounds of boost. At 3 days after the final boost, spleen cells of the immunized mice were fused with mouse myeloma cell P3X63Ag8.653 (CRL-1580; ATCC, Manassas, VA). Growing hybridoma clones were screened by enzyme-linked immunosorbent assay for binding to human PTGR2, as well as to mouse PTGR2. Antibody from hybridoma clone P9/G11-17 was used.

\section{Western Blot Analysis}

Whole-cell lysates were prepared with radioimmunoprecipitation assay buffer (BioVision, Mountain View, CA) containing protease and phosphatase inhibitor cocktail tablets (Roche Applied Science, Indianapolis, IN). Equivalent amounts of protein were resolved by SDS-PAGE and transferred to polyvinylidene fluoride microporous membrane (Millipore, Billerica, MA). Nonspecific antibody binding sites were blocked with $5 \%$ nonfat milk in PBS containing $0.1 \%$ Tween 20 (PBST), and membranes were probed with the following antibodies: PTGR2, Hsp70 (GeneTex, Irvine, CA), Bax (Millipore), Bcl-2, caspase-3 (pro) and caspase-3 (active) (Epitomics, Burlingame, CA), p44/42 MAPK (ERK1/2) (137F5), and phospho-p44/42 MAPK (ERK1/2) (Thr202/Tyr204) (D13.14.4E) XP (Cell Signaling Technology, Danvers, MA). Secondary antibodies were conjugated to horseradish peroxidase or alkaline phosphatase (Santa Cruz Biotechnology, Santa Cruz, CA), and peroxidase activity or phosphatase activity was visualized using chemiluminescent horseradish peroxidase substrate (Millipore) or BCIP/NBT phosphatase substrate (KPL, Gaithersburg, MD), respectively.

\section{Quantification of 13,14-Dihydro-15-Keto-PGE Production}

Equal numbers of cells were seeded in 24-well plates, and the culture medium was collected to measure the concentration of 13,14-dihydro-15-keto- $\mathrm{PGE}_{2}$ using a 
prostaglandin E metabolite enzyme immunoassay kit according to the manufacturer's instructions (Cayman Chemical, Ann Arbor, MI). The absorbance was measured using a microtiter culture plate reader at wavelength $405 \mathrm{~nm}$. The ratio of the absorbance of standard wells to that of the maximum binding well $\left(\% B / B_{0}\right)$ versus PGEM concentration was plotted, and the equation $y=m x+b$ from the standard curve was used for obtaining the concentration for each sample. The concentration for the control samples was set as 1 , and the relative levels of 13,14-dihydro-15-keto-PGE in PTGR2-knockdown or PTGR2-overexpressing cells were calculated relative to the controls.

\section{Cell Proliferation Assay}

Cell proliferation was measured by MTS assay (CellTiter 96 aqueous one solution cell proliferation assay) (Promega, Madison, WI) according to the manufacturer's instructions. Briefly, $1 \times 10^{3}$ cells were seeded in each well of 96-well microtiter plates. At the indicated time points ( 1 to 6 days after seeding), the percentage of viable cells were quantified by measuring the absorbance at $490 \mathrm{~nm}$ using a microtiter culture plate reader. The absorbance at $490 \mathrm{~nm}$ for day 1 was set as 1 , and the relative proliferation rate for each day was calculated relative to day 1 .

\section{Soft Agar Colony Formation Assay}

Using a hemocytometer, $5 \times 10^{3}$ lentiviral-transduced AGS gastric cancer cells were counted and were suspended in $0.6 \%$ agarose (SeaPlaque low-temperature agarose; Lonza, Rockland, ME) with RPMI 1640 medium containing $10 \%$ fetal bovine serum and plated into six-well plates on top of an existing layer of $0.8 \%$ base agarose prepared with the same medium. After the gel had solidified, $0.5 \mathrm{~mL}$ medium was placed on top of the upper agarose layer. The plates were incubated at $37^{\circ} \mathrm{C}$ in a $5 \% \mathrm{CO}_{2}$ incubator. After 14 days, colonies were stained with $0.05 \%$ crystal violet for 30 minutes and then were photographed. The numbers of colonies were quantified using GeneTools software version 4.01 (SynGene, Frederick, MD).

\section{Xenograft Tumor Models}

The nude mouse (BALB/C-nu/nu) xenografts were established by subcutaneously inoculating $1 \times 10^{7}$ lentiviraltransduced control $(n=5)$ or PTGR2-knockdown $(n=7)$ AGS cells or control $(n=6)$ or PTGR2-overexpressing $(n=5)$ AGS cells that were mixed with equal volume of Matrigel matrix (BD Biosciences, San Jose, CA) into the flanks of 4-week-old female nude mice. The sizes of the resulting tumors were measured with calipers; volume was calculated as $\pi / 6 \times$ length $\times(\text { width })^{2}$. All animal experiments were performed in accordance with protocols approved by the National Taiwan University Institutional Animal Care and Use Committee.

\section{Flow Cytometric Cell Cycle and Cell Death Analysis}

To analyze cell cycle, cells (control or 24-hour serum starvation) were collected and fixed with ice-cold $70 \%$ ethanol in PBS and stored at $-20^{\circ} \mathrm{C}$ until use. Cells were resuspended in PBS containing $3 \mu \mathrm{g} / \mathrm{mL}$ RNase A (Qiagen, Valencia, CA) and $50 \mu \mathrm{g} / \mathrm{mL}$ propidium iodide (Sigma-Aldrich, St. Louis, MO) and were incubated at $37^{\circ} \mathrm{C}$ in the dark for 30 minutes before flow cytometric analysis. Flow cytometry was performed on a FACSCalibur system (BD Biosciences). Apoptosis was detected using a Cayman Chemical multi-parameter apoptosis assay kit according to the manufacturer's instructions and was analyzed using FL1 (annexin V-fluorescein isothiocyanate) and FL3 [7-aminoactinomycin $D(7-A A D)]$ detectors. Annexin V-positive cells were defined as apoptotic, and annexin V-7-AAD-double positive cells were defined as necrotic.

\section{Assessment of OCR and ECAR}

The metabolic activities of the cells were determined by measuring the oxygen consumption rate (OCR; indicative of mitochondrial oxidative respiration) and the extracellular acidification rate (ECAR; a measure of extracellular energy production indicative of glycolysis), using an XF24 extracellular flux analyzer (Seahorse Bioscience, North Billerica, MA) as described previously. ${ }^{26}$

\section{Measurement of ROS Production}

Cells without treatment or after treatment with dimethyl sulfoxide control or $5 \mathrm{mmol} / \mathrm{L} \mathrm{N}$-acetyl-L-cysteine (NAC) (Sigma-Aldrich) overnight were harvested in PBS and incubated in the presence of $10 \mu \mathrm{mol} / \mathrm{L} \mathrm{2}^{\prime} 7^{\prime}$-dichlorodihydrofluorescein diacetate $\left(\mathrm{H}_{2} \mathrm{DCFDA}\right)$ (Life Technologies-Invitrogen) in the dark at $37^{\circ} \mathrm{C}$ for 30 minutes before analysis on a FACSCalibur flow cytometry system (BD Biosciences) using the FL-1 detector. ROS production was determined from histogram data using CellQuest software version 3.3 (BD Biosciences).

\section{Quantitative Real-Time PCR}

Quantitative real-time PCR analysis was performed as described previously, using the comparative cycle threshold method $\left(2^{-\Delta \Delta \mathrm{Ct}}\right)$ to quantify gene expression. ${ }^{29,30}$ The mRNA levels were normalized to human cyclophilin expression (sense primer: 5'-GCATACGGGTCCTGGCATCTTGTCC-3' and antisense primer: 5'-ATGGTGATCTTCTTGCTGGTCTTGC-3'). The sequences for the specific sets of primers for Bax and $\mathrm{Bcl}-2$ were as previously reported. ${ }^{31}$

\section{Human Tissue Immunopathological Studies and Clinical Outcomes}

From 1995 to 1997, 52 patients with gastric carcinoma who received surgical gastrectomy, detailed pathological assessment, and regular follow-up at the National Taiwan University Hospital were recruited for the present study. Clinical stage was defined according to the criteria of American Joint Committee on Cancer (AJCC; 6th edition, 2002). Patient follow-up after the surgery averaged 8.75 years. Patient characteristics are summarized in Table 1. The present study was conducted according to regula- 
Table 1. Clinical Characteristics of Patients with Gastric Cancers

\begin{tabular}{ll}
\hline \multicolumn{1}{c}{ Characteristic } & Value (no.) \\
\hline Age (average years \pm SD) & $63 \pm 11.2$ \\
Male/female & $35: 20$ \\
Histology type I, D, O & $25: 28: 1$ \\
Cancer stage 1, 2, 3, 4 & $16: 14: 23: 2$ \\
Tumor size T1, T2, T3, T4 & $7: 24: 23: 1$ \\
Lymph node status N0, N1, N2, N3 & $19: 23: 12: 1$ \\
\hline
\end{tabular}

$\mathrm{D}$, diffuse type; I, intestinal type; $\mathrm{O}$, other.

tions of the Institutional Review Board of the National Taiwan University Hospital; the specimens were rendered anonymous and were analyzed in a masked manner.

Immunohistochemistry was performed as described previously, using the avidin-biotin complex immunoperoxidase method. ${ }^{32}$ Briefly, sections (5 $\mu \mathrm{m}$ thick) from formalin-fixed, paraffin-embedded tumor specimens were prepared; immunohistochemical staining was performed using mouse monoclonal antibody against human PTGR2 or nonimmune IgG, and specimens were examined under a bright-field microscope. PTGR2 staining positivity was meticulously examined by a pathologist (C.T.S.) and was classified into grades $1,2,3$, and 4 (from lower to higher stain intensity) (Table 2). For the negative control, primary antibody was replaced by normal saline.

\section{Chemosensitivity Assay}

For chemosensitivity assay, $5 \times 10^{3}$ cells were counted and seeded in medium containing $5,10,25$, or $50 \mu \mathrm{mol} / \mathrm{L}$ of cisplatin [cis-diammineplatinum(II) dichloride] or 5-fluorouracil (Sigma-Aldrich). After 24 hours of incubation, chemosensitivities were analyzed using an MTS assay (Promega) according to the manufacturer's instructions, measuring the absorbance at $490 \mathrm{~nm}$. The absorbance at $490 \mathrm{~nm}$ for cells without drug treatment was set as 100 . The percent survival for cells treated with drugs was calculated in terms of absorbance relative to cells without drug treatment.

\section{Statistical Analysis}

For the clinical statistical analysis, data are expressed as means \pm SD. PTGR2 immunopathological stain intensity between tumor and nontumor portions of gastric cancer tissue was compared using the nonparametric Wilcoxon matched pairs test. The Kaplan-Meier method was used to estimate the survival function of patients and the log-rank test was used to compare survival of patients differing in tumor-portion PTGR2 stain intensity. Cox proportional hazard regression was used to analyze the association of tumor-portion PTGR2 stain intensity with survival adjusted for other clinical prognostic factors. Statistical analysis was performed using SAS 8.0 software (SAS Institute, Cary, NC). For all other experiments, data are expressed as means \pm SE and are based on more than three independent experiments. Groups were compared by Student's t-test. Statistical analyses were performed with GraphPad Prism 4 (GraphPad Software, La Jolla, CA). For all comparisons, $P \leq 0.05$ was used as the criterion of significance.

\section{Results}

\section{PTGR2 Levels Affect the Oncogenic Potency of Gastric Cancer Cells in Vitro and in Vivo}

To determine the role of PTGR2 in gastric cancer, a lentiviral-based shRNA approach was used to knock down or to overexpress PTGR2 in AGS and SNU-16 gastric cancer cell lines (Figure 1, A and B). Because PTGR2 catalyzes the reduction of 15-keto-PGE 2 into 13,14-dihydro-15-keto-PGE ${ }_{2}{ }^{13,14}$ we performed further quantification and showed that PTGR2 knockdown resulted in reduced production of 13,14-dihydro-15-keto-PGE ${ }_{2}$, whereas PTGR2 overexpression resulted in elevated production (Figure 1, C and D). In vitro proliferation analysis showed that PTGR2 knockdown greatly suppressed and PTGR2 overexpression increased the proliferation rate of AGS (Figure 1E) and SNU-16 (Figure 1F) gastric cancer cell lines. Similar results were obtained using different shRNAi targeting different sequence of PTGR2 (data not shown). Furthermore, PTGR2 knockdown resulted in substantial reduction in the number of colonies formed, whereas PTGR2 overexpression greatly increased the transformation potential of the cells, as judged by the increased colony numbers (Figure 2, A and B).

To confirm the oncogenic potential of PTGR2 and to ascertain it in vivo, we subcutaneously inoculated nude mice with PTGR2-knockdown or PTGR2-overexpressing AGS cells. PTGR2-knockdown cells showed reduced tumorigenicity in vivo, as indicated by the significantly smaller sizes of the tumors developed (Figure 2C), whereas mice receiving PTGR2-overexpressing cells showed enhanced tumorigenicity in vivo (Figure 2D). These results showed that suppression of PTGR2 could negatively influence the tumorigenic potential of gastric cancer cells.

\section{PTGR2 Affects Gastric Cancer Cell Growth by Altering the Susceptibility toward Cell Death}

To investigate the mechanisms through which PTGR2 takes part in gastric cancer, we first performed cell cycle analysis to clarify the cause for the modulating effect of PTGR2 on growth rate. The observed effects of PTGR2 knockdown on cell proliferation were not associated with alterations in driving cell cycle progression, because no significant differences were observed in the distribution of cell populations in G1, S, or G2/M phases. However, accu-

Table 2. PTGR2 Immunopathological Stain Intensity between Tumor Portion and Nontumor Portion of Gastric Cancer

\begin{tabular}{llll}
\hline & \multicolumn{2}{c}{$\begin{array}{c}\text { PTGR2 Stain intensity } \\
\text { (no.) }\end{array}$} & \\
\cline { 2 - 3 } Group & Nontumor & Tumor & P value \\
\hline All patients & $43: 5: 1: 0$ & $8: 11: 10: 23$ & $<0.0001$ \\
Intestinal type & $20: 1: 1: 0$ & $3: 5: 4: 12$ & $<0.0001$ \\
Diffuse type & $21: 4: 0: 0$ & $5: 6: 5: 10$ & $<0.001$ \\
\hline
\end{tabular}

Data are expressed as the frequency distribution of samples with grade 1, 2, 3, and 4 PTGR2 stain, from lower to higher intensity of staining. 
A PSGR2

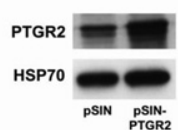

$\mathrm{C}$

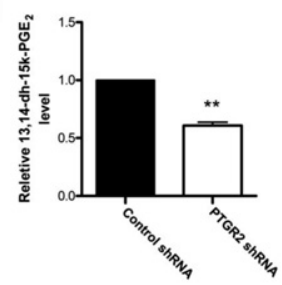

E

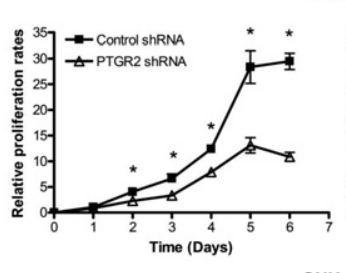

$\mathrm{F}$

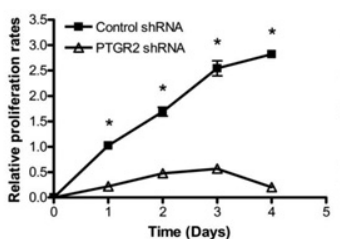

SNU-16
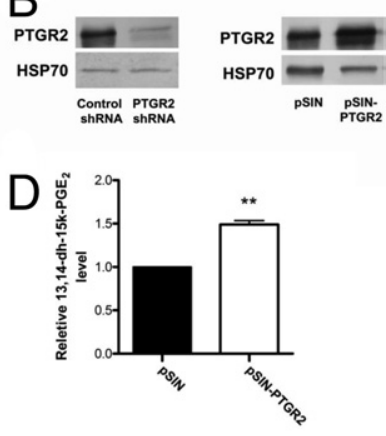

AGS
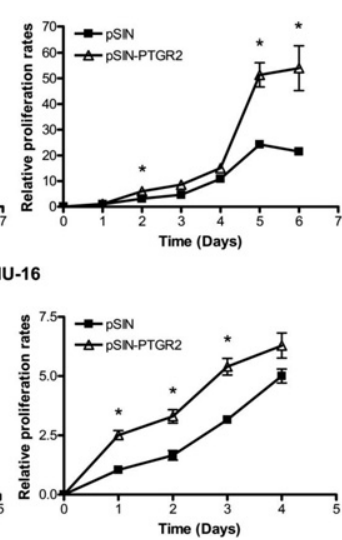

Figure 1. PTGR2 levels affect gastric cancer cell proliferation. $\mathbf{A}$ and $\mathbf{B}$ : Efficiency of lentiviral shRNA-mediated PTGR2 knockdown and overexpression. PTGR2 protein expression in AGS (A) and SNU-16 (B) cells was detected by Western blot analysis. HSP70 served as loading control. C and D: Relative production of 13,14-dihydro-15-keto-PGE $\mathrm{PG}_{2}$ in PTGR2-knockdown (C) or PTGR2-overexpressing (D) AGS cells, compared with control cells. The concentration for the control samples was set as 1 , and the relative levels of 13,14-dihydro-15-keto-PGE 2 in PTGR2-knockdown or PTGR2-overexpressing cells were presented as values relative to the control. $\mathbf{E}$ and $\mathbf{F}$ : Relative proliferation rates of PTGR2 knockdown or overexpression in AGS (E) and SNU-16 (F) cells. Cell proliferation was evaluated by MTS assay at the indicated time points. Data are expressed as means \pm SE. ${ }^{*} P<0.05,{ }^{* *} P<$ 0.01 , Student's $t$-test. $n=3$.

mulations of sub-G1 cells were induced (Figure 3A). Because this population contains dead cells, we further verified the role of PTGR2 in apoptosis or necrosis by annexin V-7AAD binding assay. PTGR2 knockdown indeed induced cell death, with significant accumulation in the percentage of both apoptotic and necrotic cells (Figure 3B), whereas PTGR2 overexpression rendered cells less prone to apoptosis and necrosis (Figure $3 C$ ). These findings prompted the question of what role PTGR2 has in mitochondrial function, because mitochondrial dysfunction is frequently the leading cause of both apoptotic and necrotic cell death. ${ }^{18}$

\section{PTGR2 Affects Cell Death by Modulating Mitochondrial Function and ROS Production}

Because metabolic transformation is a distinct feature of cancer cells and because activation of the cell death pathway is tightly linked to the regulation of mitochondrial function, $24,25,33$ we first measured the flux of oxygen [oxygen consumption rates (OCR)] and of protons [extracellular acidification rates (ECAR)] to detect changes in the rate of mitochondrial respiration and glycolysis, using extracellular flux technology. ${ }^{26}$ Over the course of 3 days, we found that PTGR2 knockdown resulted in a lower ECAR/OCR ratio, compared with the control cell line (Figure $4 \mathrm{~A}$ ), indicating induced oxidative phosphorylation leading to increased utilization of mitochondrial respiration. On the other hand, the more oncogenic PTGR2-overexpressing cell line exhibited a significantly higher ECAR/OCR ratio (Figure $4 B$ ), indicating increased utilization of glycolysis, which is a metabolic characteristic of tumors. ${ }^{34}$

The production of ROS is a physiological process dependent on the cellular activity of mitochondrial respiration, and induced oxidative phosphorylation should generate more ROS by-products. ${ }^{18,35}$ Under normal cellular condition, elevated ROS production was observed in PTGR2-knockdown cells (Figure 4C) and reduced ROS production was observed in PTGR2-overexpressing cells (Figure 4D). This is consistent with the finding showing that the apparently more oncogenic PTGR2-overexpressing cells relied less on the ROS-generating aerobic respiration. We further treated cells with antioxidant NAC to eliminate the excess ROS produced in PTGR2-knockdown cells. After NAC treatment, PTGR2-knockdown
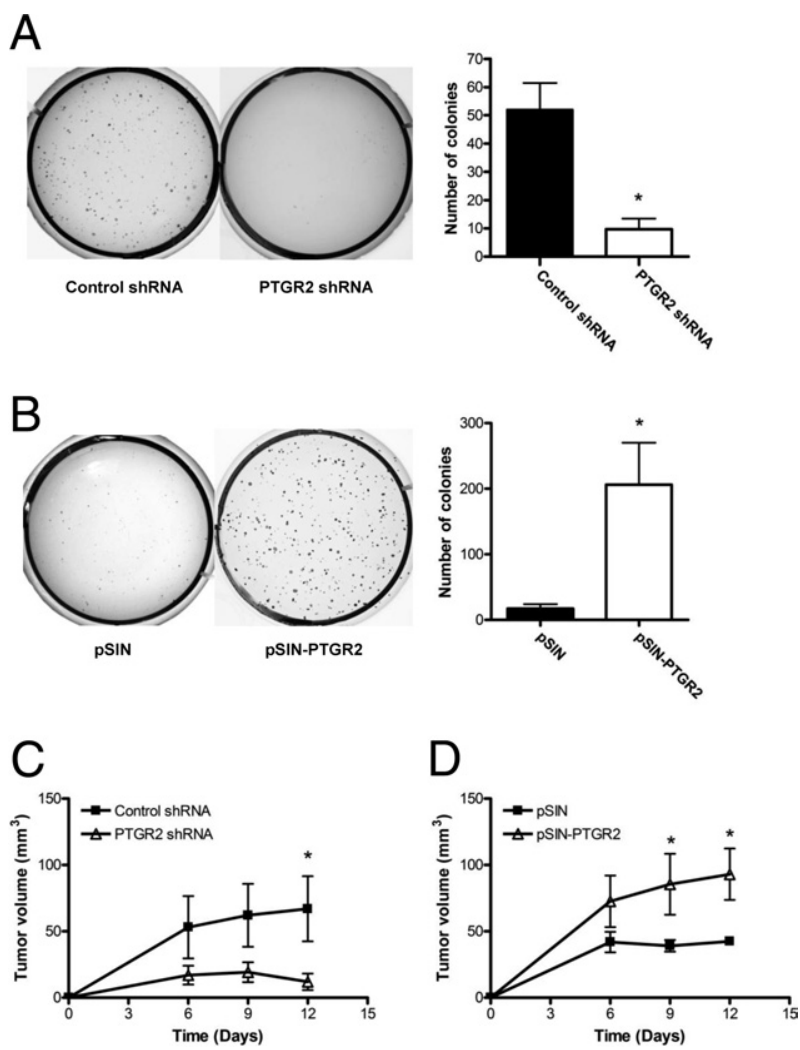

Figure 2. Oncogenic potency of PTGR2 in vitro and in vivo. $\mathbf{A}$ and $\mathbf{B}$ Images of colony growth and numbers of colonies formed in soft agar assay of PTGR2-knockdown (A) and PTGR2-overexpressing (B) AGS cells, compared with controls. Colonies were stained with $0.05 \%$ crystal violet and photographed and counted after 14 days. C and D: Down-regulation of PTGR2 reduced tumorigenicity of AGS cells and overexpression of PTGR2 enhanced tumorigenicity of AGS cells in nude mice. Tumor volumes in mice receiving PTGR2-knockdown (C) and PTGR2-overexpressing (D) AGS cells were monitored over the course of the experiment. Data are expressed as means \pm SE. ${ }^{*} P<0.05$, Student's $t$-test. $n=3$ (A and $\left.\mathbf{B}\right) ; n=5$ (C) control AGS cells; D, PTGR2-overexpressing AGS cells); $n=6$ (D, control AGS cells); $n=7$ (C, PTGR2-knockdown AGS cells). 

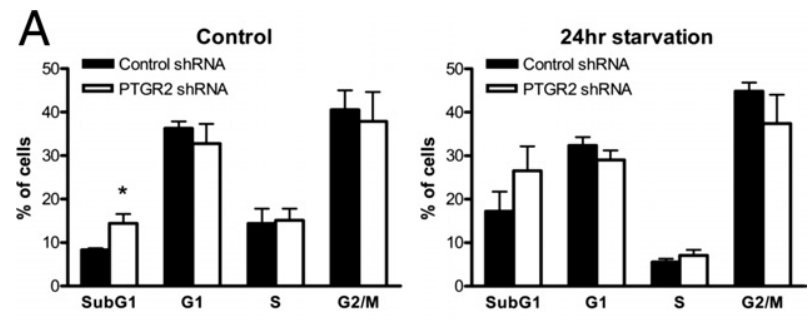

B
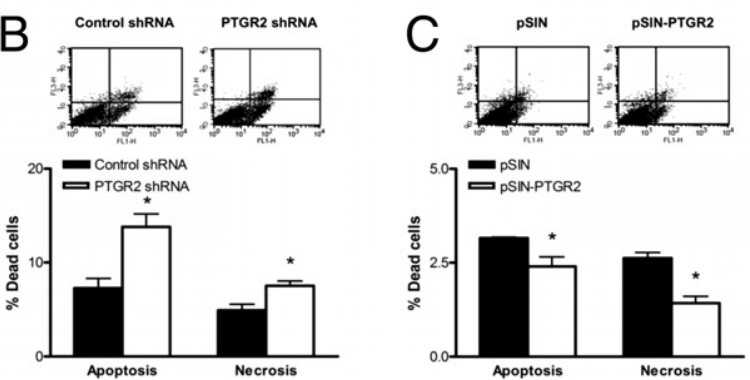

Figure 3. Effect of PTGR2 on the susceptibility of gastric cancer cells to cell death. A: Distribution of cell cycle phases in PTGR2-knockdown versus control AGS cells under normal growth conditions (control) or after 24 hours of serum starvation. Cells were stained with propidium iodide and analyzed by flow cytometry. B and C: The percentage of dead cells was evaluated by annexin $\mathrm{V}$ and 7-AAD staining of PTGR2-knockdown (B) and PTGR2-overexpressing (C) AGS cells, compared with control cells. The flow cytometry plots show annexin V-FITC binding (FL1-H) and 7-AAD staining (FL3-H). The bar graphs distinguish dead cells as apoptotic or necrotic cells. Data are expressed as means $\pm \mathrm{SE}$. ${ }^{*} P<0.05$ Student's $t$-test. $n=3$.

cells produced levels of ROS similar to those in control cells (Figure 5A). Moreover, elimination of ROS significantly reduced the percentage of dead cells in PTGR2knockdown cells (Figure 5C). In control cells, however, the effect of cell death prevention after ROS elimination was not observed (Figure 5B). Taken together, our results suggest that PTGR2 affects cell death by modulating the mitochondria-ROS-dependent pathway.

\section{PTGR2 Modulates Mitochondrial Apoptotic} Signaling through Both the ERK1/2-Caspase 3 Pathway and Bcl-2-Family Proteins

Extracellular signal-regulated protein kinases 1 and 2 (ERK1/2)-specific phosphatases are sensitive to ROS, and ROS-induced ERK1/2-mediated cell death is associated with the intrinsic apoptotic pathway characterized by the activation of caspase $3 .^{23,36-38}$ We therefore investigated further and found that induced ERK1/2 activation followed by the activation of caspase 3 activities (indicated by the induction of ERK $1 / 2$ phosphorylation and the cleavage of caspase 3) was observed in PTGR2knockdown cells. In contrast, PTGR2-overexpressing cells exhibited suppressed ERK $1 / 2$ and caspase 3 activities (Figure 6A). Another major mitochondria-mediated apoptotic pathway involves Bcl-2 family proteins. We therefore also investigated the possible involvement of PTGR2 in regulating Bcl-2 family protein expression. Indeed, quantitative real-time PCR and Western blotting analysis showed induced proapoptotic Bax and suppressed $\mathrm{Bcl}-2$ oncogene expression in PTGR2-knockdown cells (Figure 6, B and C), whereas PTGR2-overex- pressing cells exhibited elevated Bcl-2 expression and reduced Bax protein expression (Figure 6D). Our data indicate that the apoptotic pathway induced by the loss of PTGR2 is mediated through its effect on mitochondrial function, followed by activation of the ERK1/2-caspase 3 pathway and modulation of $\mathrm{Bcl}-2$ family proteins.

\section{Clinical Significance and Immunopathological Analysis of PTGR2 Expression in Human Gastric Cancer Tissues}

To validate clinical relevance of PTGR2 in gastric cancer, tumor tissue sections from patients with gastric cancer were subjected to immunohistochemical staining for PTGR2 expression. PTGR2 staining was significantly stronger in the tumor portion of the tissue, compared with adjacent nontumor portions (Figure 7; Table 2). Moreover, higher PTGR2 stain intensity in the tumor portion was significantly associated with lower survival in pa-
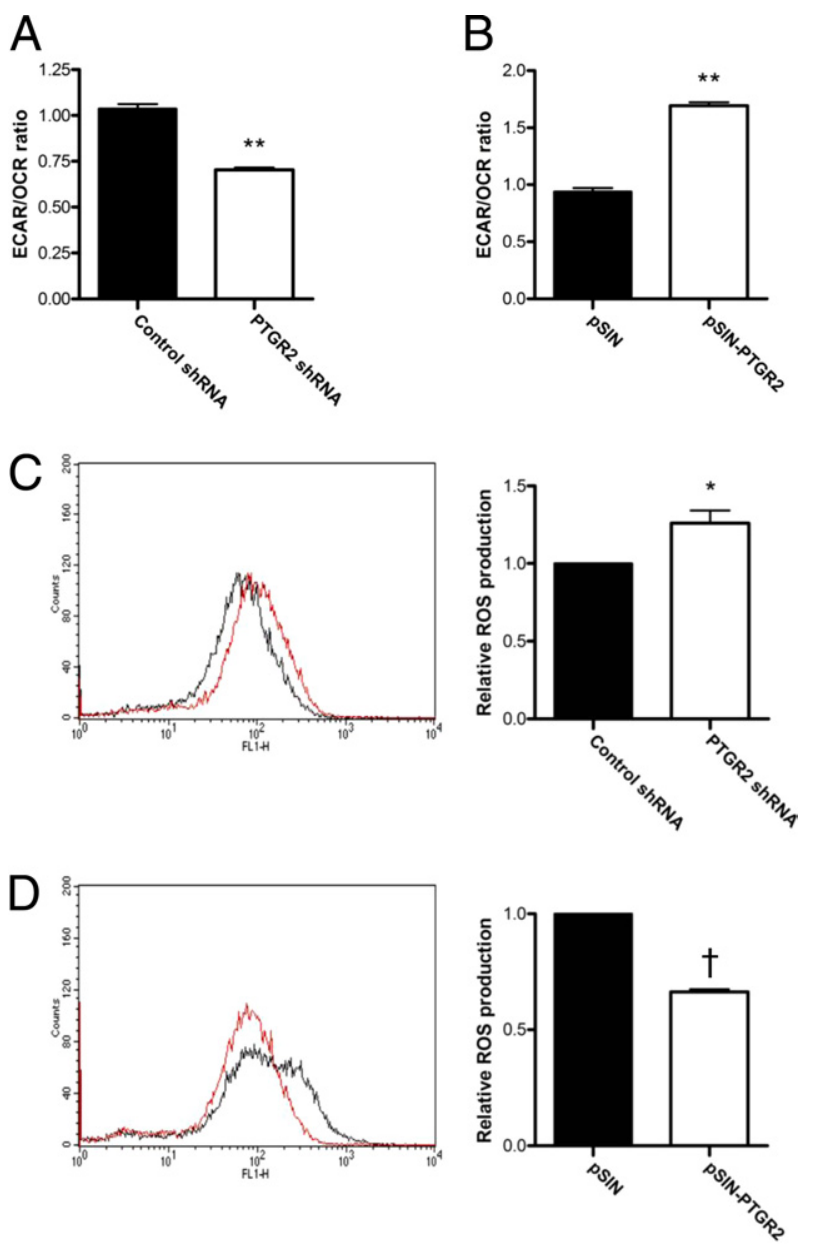

Figure 4. PTGR2 modulates mitochondrial function and ROS production. A and B: Ratio of extracellular acidification rate (ECAR) to oxygen consumption rate (OCR) of PTGR2-knockdown (A) and PTGR2-overexpressing (B) AGS cells, compared with control cells, on day 3 of cultivation. $\mathbf{C}$ and $\mathbf{D}$ : ROS production in PTGR2-knockdown (C, red profile) or PTGR2-overexpressing (D, red profile) AGS cells, compared with control cells (black profile), was detected using $\mathrm{H}_{2} \mathrm{DCF}$ dye and flow cytometry. Data are expressed as means \pm SE. Data represent one of three independent experiments with similar results. ${ }^{*} P<0.05,{ }^{* *} P<0.01$, and ${ }^{\dagger} P<0.005$ Student's $t$-test. $n=6$. 
A

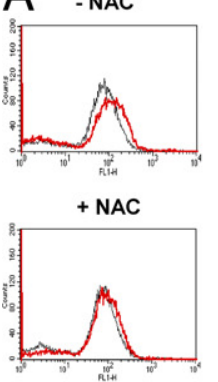

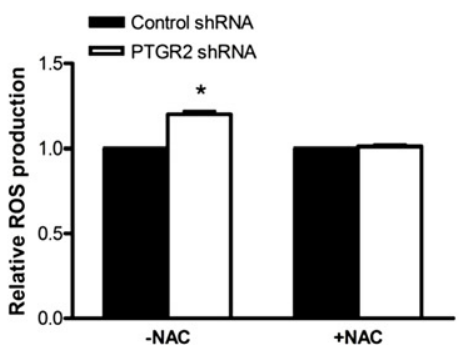

B

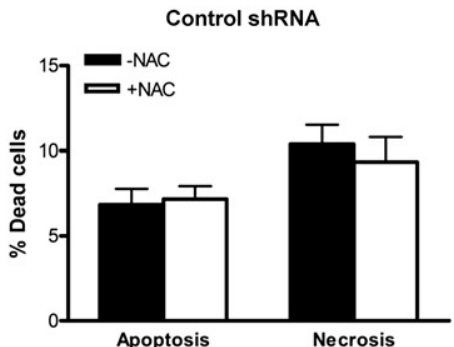

C

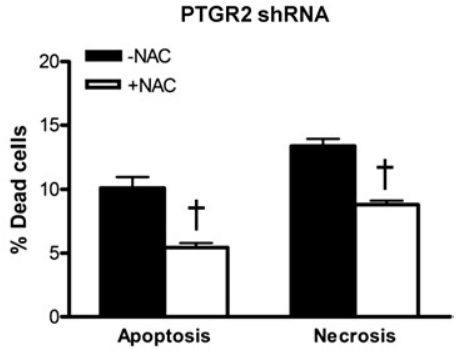

Figure 5. Elimination of ROS prevents cell death induced in PTGR2-knockdown cells. A: ROS production in PTGR2-knockdown versus control AGS cells under treatment with dimethyl sulfoxide control (-NAC) or after treatment with $5 \mathrm{mmol} / \mathrm{L}$ NAC (+NAC) overnight. ROS production was detected using $\mathrm{H}_{2} \mathrm{DCF}$ dye and flow cytometry. The black peak represents control cells and the red peak represents PTGR2-knockdown cells. B and C: The percentage of dead cells as apoptotic or necrotic was evaluated by annexin $\mathrm{V}$ and 7-AAD staining of control (B) and PTGR2-knockdown (C) AGS cells under treatment with dimethyl sulfoxide control $(-\mathrm{NAC})$ or $5 \mathrm{mmol} / \mathrm{L} \mathrm{NAC}(+\mathrm{NAC})$ overnight. Data are expressed as means $\pm \mathrm{SE} .{ }^{*} P<0.05,{ }^{\dagger} P<0.005$ Student's $t$-test. $n=3(\mathbf{A}) ; n=4(\mathbf{B}$ and $\mathbf{C})$.

tients with intestinal-type gastric cancer $(P=0.03$ logrank test) (Figure 8 ). The association remained significant after adjustment for other clinical predictors (hazard ratio per stain grade, 2.47 [95\% confidence interval 1.27 to

A
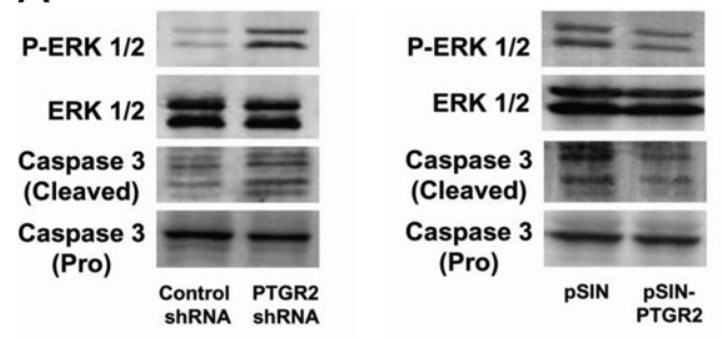

B Bcl-2
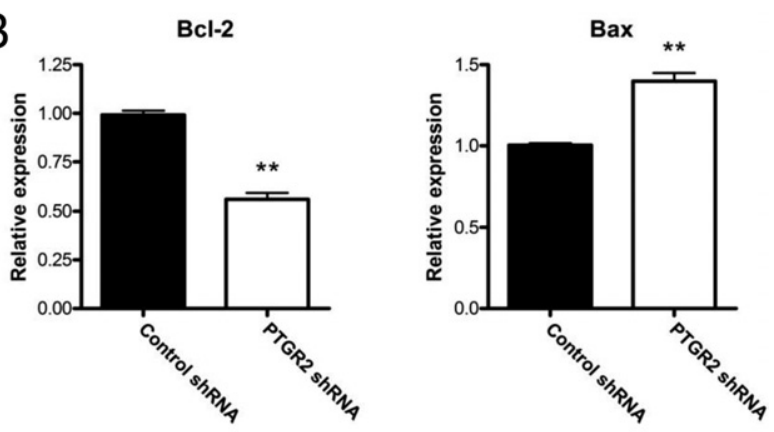

C

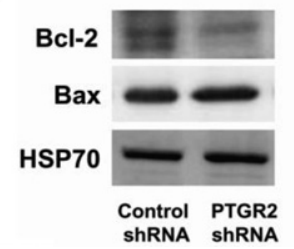

D

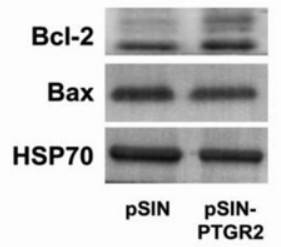

Figure 6. Knockdown of PTGR2 induced ERK1/2-caspase 3-mediated apoptotic signaling and altered $\mathrm{Bcl}-2$ family protein expression. A: Western blot analysis of expression levels of ERK1/2 phosphorylation and caspase 3 cleavage of PTGR2-knockdown (left) or PTGR2-overexpressing (right) AGS cells. B: Expression levels of Bcl-2 and Bax in PTGR2-knockdown AGS cells as measured by quantitative real-time PCR. The mRNA levels were normalized to human cyclophilin expression level. C and D: Western blot analysis of Bcl-2 and Bax protein expression in PTGR2-knockdown (C) or PTGR2overexpressing (D) AGS cells. HSP70 served as loading control. Data are expressed as means $\pm \mathrm{SE}$. ${ }^{* *} P<0.01$, Student's $t$-test. $n=3$.
4.81], $P=0.007)$. However, the association was not significant in patients with diffuse-type gastric cancer (Table 3). The negative correlation between PTGR2 expression and patient survival further supports the notion that PTGR2 is involved in gastric cancer biology.

\section{Involvement of PTGR2 in Chemotherapeutic Response}

Based on the clinical relevance of PTGR2 in gastric cancer, we further explored whether PTGR2 affects the chemosensitivity of the gastric cancer cells to two commonly used chemotherapeutic drugs, cisplatin and 5-fluorouracil. For both drugs, PTGR2-knockdown cells were more susceptible to drug-induced cell death (Figure 9A), compared with overexpressing PTGR2 cells, which exhibited reduced sensitivity toward cisplatin- and 5-fluorouracil-
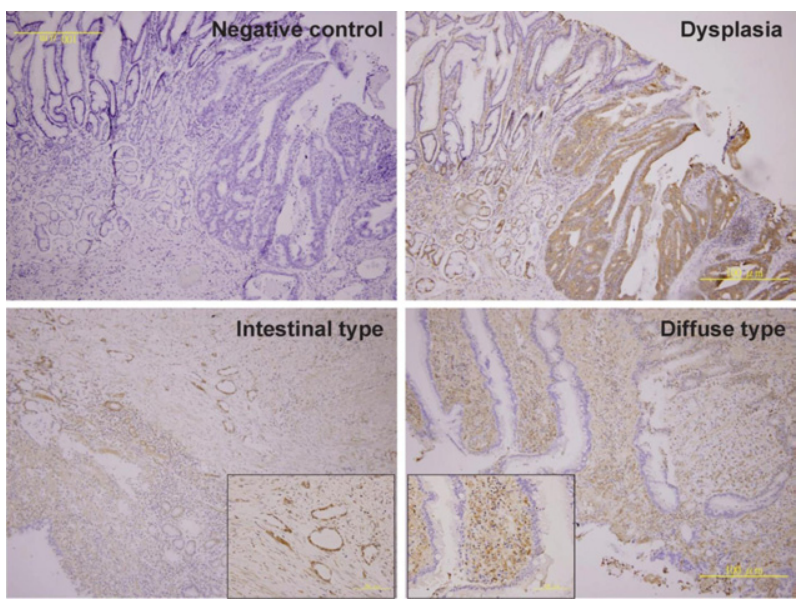

Figure 7. PTGR2 protein expression in human gastric cancer tissue. Representative tissue sections (selected from paraffin-embedded specimens of various types of gastric cancers obtained from 52 patients) for dysplasia, intestinal-type gastric cancer, and diffuse-type gastric cancer, with adjacent normal tissues. Immunohistochemical staining was performed using a specific anti-PTGR2 antibody. Tumor cells were clearly positive for PTGR2 (dark brown) in dysplastic mucosa, intestinal-type gastric cancer, and diffuse-type gastric cancer, whereas the majority of the adjacent normal tissue cells were negative for PTGR2. Insets: PTGR2-positive regions at higher magnification. Scale bar $=100 \mu \mathrm{m}$. Original magnification, $\times 100$ (main images); $\times 400$ (insets). 


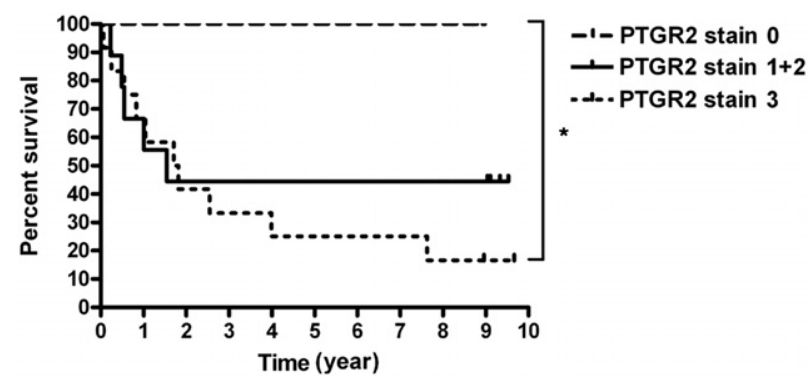

Figure 8. Relationship between PTGR2 expression and survival in patients with intestinal-type gastric cancer. Kaplan-Meier overall survival curves were plotted based on PTGR2 stain intensity. ${ }^{*} P=0.03$, log-rank test.

induced cell death (Figure 9B). This finding supports the negative correlation between PTGR2 expression and survival of gastric cancer patients from our clinical data.

\section{Discussion}

In the present study, we have demonstrated through in vitro and in vivo analysis that PTGR2 modulates the oncogenic potency of gastric cancer cells by impeding mitochondria- and ROS-mediated cell death involving both ROS-ERK1/2-caspase 3 signaling and Bcl-2-family proteins. Importantly, we have also presented clinical evidence showing the specific negative correlation between PTGR2 and survival of patients with intestinal-type gastric cancer. These findings, together with our chemosensitivity study, further substantiate the significance of PTGR2 in gastric cancer.

Previously, the only known biological role of PTGR2 was to catalyze the reduction of the natural PPAR $\gamma$ ligand 15-keto-PGE 2 into the downstream metabolite 13,14-dihydro-15-keto-PGE 2 , which in turn suppresses PPAR $\gamma$ activation. ${ }^{13,14}$ Based on the present results, we propose a novel functional importance of PTGR2 in gastric cancer and cell death execution. Various roles for prostaglandins in cancer biology have been extensively studied, especially the prostaglandin $E_{2}$ pathway. ${ }^{4,5}$ A recent genomewide association study identified the region encoding prostaglandin E receptor 4 as a novel susceptibility locus for gastric cancer. ${ }^{12}$ Thus, it is not surprising that PTGR2 plays a role in cancer biology, possibly by modulating the metabolism of 15-keto-PGE 2 . The level of 15-keto-PGE 2 is in part dependent on PTGR2 activity, and previous studies demonstrating the prevention of gastric carcinogen-

Table 3. Cox Proportional Hazards Analysis of Mortality in Patients with Intestinal-Type Gastric Cancer

\begin{tabular}{lll}
\hline \multicolumn{1}{c}{ Variable } & \multicolumn{1}{c}{$\begin{array}{c}\text { Hazard ratio } \\
(95 \% \mathrm{Cl})\end{array}$} & $P$ value \\
\hline PTGR2 stain, per grade & $2.47(1.27-4.81)$ & 0.007 \\
Age, per year & $1.13(1.04-1.23)$ & 0.003 \\
Male sex & $1.56(0.36-6.72)$ & 0.55 \\
Tumor size, per grade & $3.51(1.04-11.82)$ & 0.04 \\
Lymph-node involvement, & $1.29(1.10-1.59)$ & 0.001 \\
$\quad$ per node & & \\
\hline
\end{tabular}

$\mathrm{Cl}$, confidence interval.
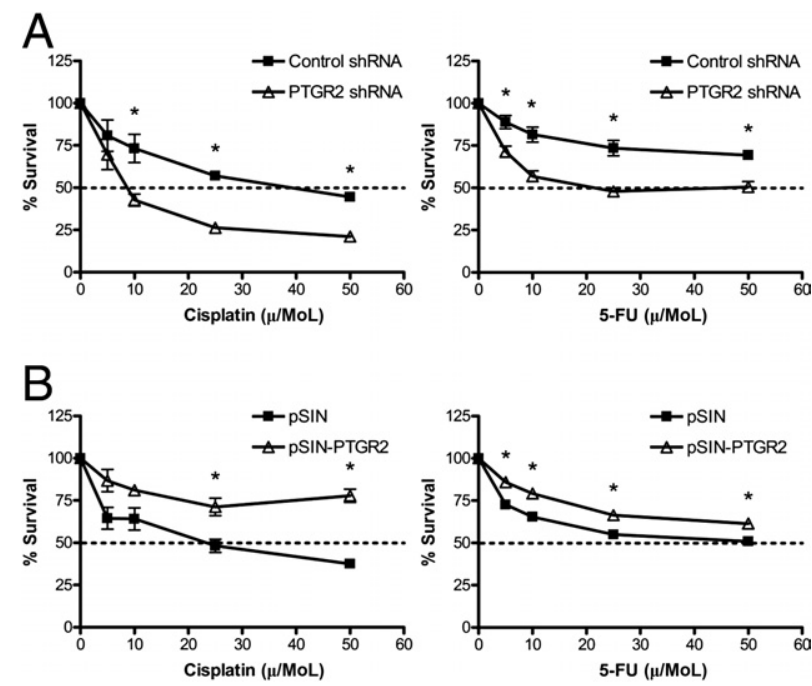

Figure 9. Knockdown PTGR2 showed enhanced sensitivity in response to cisplatin and 5-fluorouracil (5-FU). PTGR2-knockdown (A) and PTGR2-overexpressing (B) AGS cells were treated with 5, 10, 25, or $50 \mu \mathrm{mol} / \mathrm{L}$ of cisplatin or 5-fluorouracil for 24 hours. The survival rate was determined using an MTS assay. Data are expressed as means \pm SE and represent one of three independent experiments with similar results. ${ }^{*} P<0.05$, Student's $t$-test.

esis by both the anti-inflammatory protein PPAR $y$ and its ligands ${ }^{7-11}$ are in accord with the present results and provide further support for our concept. We speculate that PTGR2 knockdown results in the induction of PPAR $\gamma$ activation.

Previous studies have also demonstrated that the inflammatory microenvironment favors promotion of tumor growth. ${ }^{39-41}$ Moreover, structural study of PTGR2 demonstrated that the anti-inflammatory drug indomethacin, which induces apoptosis and exerts anti-cancer activity by inhibiting the prostaglandin synthesis enzymes, can bind to and inhibit PTGR2 activity by causing its substrate-binding pocket to become less compact. ${ }^{14,42,43}$ In light of these studies, a PTGR2-involved oncogenic pathway may be associated with PPAR $\gamma$ activation status and with changes in prostaglandin metabolism and the inflammatory microenvironment.

The significance of the present study is further established by the clinical findings showing the negative correlation of PTGR2 expression level with survival of patients with intestinal-type gastric cancer. It was peculiar that this correlation was not observed in those with diffuse-type gastric cancer, even though PTGR2 expression in both intestinal- and diffuse-type gastric cancer tumor tissue was similar. This could be explained in part by the absence or low expression level of Bcl-2 in diffuse-type gastric cancer. Our present results show that PTGR2 deficiency increases cell apoptosis through the modulation of Bcl-2 family proteins. Specifically, suppressed $\mathrm{Bcl}-2$ expression and induced Bax expression were observed in PTGR2-knockdown cells, but the opposite was observed in PTGR2-overexpressing cells. Bcl-2 expression is negatively correlated with apoptosis index in gastric cancers, as well as with the depth of invasion and lymph node metastasis. ${ }^{44-48}$ Two independent studies also found a much lower expression of Bcl-2 in diffusetype gastric cancer, compared with intestinal-type gas- 
tric cancer. ${ }^{49,50}$ Thus, differences in Bcl-2 expression may explain why the correlation between PTGR2 expression and patient survival was not observed in diffuse-type gastric cancer.

The biological effects of ROS are complex. High levels of ROS may induce oxidative stress, which inhibits cell proliferation and induces cell death. Low or intermediate levels, however, induce mutation and promote cell proliferation, leading to the induction of carcinogenesis. ${ }^{22}$ Because the level of ROS is in part dependent on mitochondrial function, decreased OCR (an indication of decreased mitochondrial respiration) has been documented as the mechanism underlying the resistance of glycolytic cancer cells to ROS-mediated apoptosis. ${ }^{35}$ Nonetheless, the requirement of oxidative phosphorylation for the execution of cell death is still a matter of debate. ${ }^{51,52}$ Our data show that the more oncogenic PTGR2-overexpressing cells exerted a higher ECAR/OCR ratio (indicative of attenuated mitochondrial respiration) and were more resistant to ROSmediated cell death, whereas PTGR2-knockdown cells, which exerted a lower OCR than the control cells (data not shown), had lower ECAR/OCR ratios, compared with control cells. Taken together, these data suggest that the mitochondrial network is associated with the efficiency of cell death execution mediated by ROS and that elevated ROS in PTGR2-knockdown cells may damage mitochondria, leading to a degradation in the efficiency of the mitochondrial respiratory chain enzymes and thus a decline in OCR.

Cancer cells overexpressing $\mathrm{Bcl}-2$ proteins could harbor induced mitochondrial oxygen consumption under basal conditions, which is in accord with our data. When exposed to excessive oxidative stress, Bcl-2 reduced mitochondrial ROS production to prevent a lethal build-up of ROS; thus, Bcl-2 also helps maintain a prooxidant environment that ameliorates survival and cancer progression. ${ }^{20,53,54}$ On the other hand, in the oncogenic PTGR2-overexpressing cells we observed suppressed Bax expression. Interestingly, Lalier et $\mathrm{al}^{15}$ proposed that both $\mathrm{PGE}_{2}$ and 15-keto-PGE $\mathrm{F}_{2}$, the substrate of PTGR2, could induce Bax activation. The elevated production of 13,14-dihydro-15-keto-PGE 2 in PTGR2-overexpressing cells is consistent with the notion that PTGR2 overexpression implies higher PTGR2 activities and therefore decreased levels of 15-keto-PGE 2 and Bax.

In addition to mitochondrial respiration and Bcl-2 family proteins, the activities of antioxidant enzymes such as manganese superoxide dismutase (MnSOD) and catalase are also important in fine tuning cellular ROS levels. By preventing excess ROS build up, antioxidant enzymes exert an inhibitory effect on apoptosis. ${ }^{55}$ Several studies have demonstrated induced expression and activity of antioxidant enzymes in gastric adenocarcinoma tissues. ${ }^{56-58}$ We therefore speculate that the more oncogenic PTGR2-overexpressing cells could also exert elevated antioxidant defense activity, leading to reduced ROS levels.

We have previously reported that Y259 is a critical conserved residue for PTGR2 activity. ${ }^{14}$ In addition to clarifying the involvement of the modulation on PPAR $\gamma$ pathway, overexpression of the PTGR2 enzymatic mutant could help decipher whether the oncogenic potency of PTGR2 is dependent on its catalytic activity. This would further substantiate the importance of the prostaglandin pathway in cancer biology. We also showed that PTGR2 affects both apoptotic and necrotic cell death (although we focused mainly on delineating the apoptotic pathway). Because ROS is a major mediator in necrotic cell death irrespective of the stimulus, ${ }^{17,59}$ we proposed that the effect of PTGR2 on necrotic cell death may be in part due also to ROS levels. Recently, necrotic cell death was proposed to be well controlled and programmed through signaling cascades like the caspase-dependent apoptosis. ${ }^{17}$ It would be interesting to explore and to clarify the necrotic cell death signaling regulated by PTGR2.

The present findings from functional and mechanistic analyses provide novel insights into the effects of PTGR2 in gastric cancer. In addition, our findings identify PTGR2 as a potential therapeutic target for the treatment of gastric cancer. Given that chemoresistance is a major obstacle in treating gastric cancer patients, research has been focusing on finding small-molecule inhibitors for chemotherapy in combination with existing anticancer drug, to enhance therapeutic efficacy. ${ }^{2}$ Because PTGR2 expression negatively correlated with survival of intestinal-type gastric cancer patients and because PTGR2 affects the chemosensitivities of gastric cancer cells, PTGR2 target-based therapy warrants further investigation and clinical evaluation.

\section{References}

1. Stoicov C, Saffari R, Cai X, Hasyagar C, Houghton J: Molecular biology of gastric cancer: Helicobacter infection and gastric adenocarcinoma: bacterial and host factors responsible for altered growth signaling. Gene 2004, 341:1-17

2. Matsuzaki T, Yashiro M, Kaizaki R, Yasuda K, Doi Y, Sawada T, Ohira M, Hirakawa K: Synergistic antiproliferative effect of mTOR inhibitors in combination with 5-fluorouracil in scirrhous gastric cancer. Cancer Sci 2009, 100:2402-2410

3. Wu B, Zhang Q, Shen W, Zhu J: Anti-proliferative and chemosensitizing effects of luteolin on human gastric cancer AGS cell line. Mol Cell Biochem 2008, 313:125-132

4. Wang D, Dubois RN: Prostaglandins and cancer. Gut 2006, 55:115122

5. Wang MT, Honn KV, Nie D: Cyclooxygenases, prostanoids, and tumor progression. Cancer Metastasis Rev 2007, 26:525-534

6. Fulzele SV, Chatterjee A, Shaik MS, Jackson T, Ichite N, Singh M: 15-Deoxy-Delta12,14-prostaglandin J2 enhances docetaxel anti-tumor activity against A549 and H460 non-small-cell lung cancer cell lines and xenograft tumors. Anticancer Drugs 2007, 18:65-78

7. Takahashi N, Okumura T, Motomura W, Fujimoto Y, Kawabata I, Kohgo Y: Activation of PPARgamma inhibits cell growth and induces apoptosis in human gastric cancer cells. FEBS Lett 1999, 455:135139

8. Leung WK, Bai AH, Chan VY, Yu J, Chan MW, To KF, Wu JR, Chan KK, Fu YG, Chan FK, Sung JJ: Effect of peroxisome proliferator activated receptor gamma ligands on growth and gene expression profiles of gastric cancer cells. Gut 2004, 53:331-338

9. Konturek PC, Kania J, Kukharsky V, Raithel M, Ocker M, Rembiasz K Hahn EG, Konturek SJ: Implication of peroxisome proliferator-activated receptor gamma and proinflammatory cytokines in gastric carcinogenesis: link to Helicobacter pylori-infection. J Pharmacol Sci 2004, 96:134-143

10. Chen $Y X$, Zhong $X Y$, Qin YF, Bing W, He LZ: 15d-PGJ2 inhibits cell growth and induces apoptosis of MCG-803 human gastric cancer cell line. World J Gastroenterol 2003, 9:2149-2153 
11. Ma XM, Yu H, Huai N: Peroxisome proliferator-activated receptorgamma is essential in the pathogenesis of gastric carcinoma. World J Gastroenterol 2009, 15:3874-3883

12. Shi Y, Hu Z, Wu C, Dai J, Li H, Dong J, Wang M, Miao X, Zhou Y, Lu $F$, Zhang $H$, Hu L, Jiang $Y$, Li Z, Chu M, Ma H, Chen J, Jin G, Tan W, Wu T, Zhang Z, Lin D, Shen $\mathrm{H}$ : A genome-wide association study identifies new susceptibility loci for non-cardia gastric cancer at 3q13.31 and 5p13.1. Nat Genet 2011, 43:1215-1218

13. Chou WL, Chuang LM, Chou CC, Wang AH, Lawson JA, FitzGerald GA, Chang ZF: Identification of a novel prostaglandin reductase reveals the involvement of prostaglandin E2 catabolism in regulation of peroxisome proliferator-activated receptor gamma activation. J Biol Chem 2007, 282:18162-18172

14. Wu YH, Ko TP, Guo RT, Hu SM, Chuang LM, Wang AH: Structural basis for catalytic and inhibitory mechanisms of human prostaglandin reductase PTGR2. Structure 2008, 16:1714-1723

15. Lalier L, Cartron PF, Olivier C, Loge C, Bougras G, Robert JM, Oliver $\mathrm{L}$, Vallette FM: Prostaglandins antagonistically control Bax activation during apoptosis. Cell Death Differ 2011, 18:528-537

16. Kakkar $P$, Singh BK: Mitochondria: a hub of redox activities and cellular distress control. Mol Cell Biochem 2007, 305:235-253

17. Festjens N, Vanden Berghe T, Vandenabeele P: Necrosis, a wellorchestrated form of cell demise: signalling cascades, important mediators and concomitant immune response. Biochim Biophys Acta 2006, 1757:1371-1387

18. Borutaite V: Mitochondria as decision-makers in cell death. Environ Mol Mutagen 2010, 51:406-416

19. Kroemer G, Pouyssegur J: Tumor cell metabolism: cancer's Achilles heel. Cancer Cell 2008, 13:472-482

20. Krishna S, Low IC, Pervaiz S: Regulation of mitochondrial metabolism: yet another facet in the biology of the oncoprotein $\mathrm{Bcl}-2$. Biochem $\mathrm{J}$ 2011, 435:545-551

21. Curtin JF, Donovan M, Cotter TG: Regulation and measurement of oxidative stress in apoptosis. J Immunol Methods 2002, 265:49-72

22. Fang J, Seki $\mathrm{T}$, Maeda $\mathrm{H}$ : Therapeutic strategies by modulating oxygen stress in cancer and inflammation. Adv Drug Deliv Rev 2009, 61:290-302

23. Cagnol S, Chambard JC: ERK and cell death: mechanisms of ERKinduced cell death-apoptosis, autophagy and senescence. FEBS J 2010, 277:2-21

24. Orrenius S: Reactive oxygen species in mitochondria-mediated cell death. Drug Metab Rev 2007, 39:443-455

25. Lemasters JJ, Qian T, Bradham CA, Brenner DA, Cascio WE, Trost LC, Nishimura Y, Nieminen AL, Herman B: Mitochondrial dysfunction in the pathogenesis of necrotic and apoptotic cell death. J Bioenerg Biomembr 1999, 31:305-319

26. Wu M, Neilson A, Swift AL, Moran R, Tamagnine J, Parslow D, Armistead S, Lemire K, Orrell J, Teich J, Chomicz S, Ferrick DA: Multiparameter metabolic analysis reveals a close link between attenuated mitochondrial bioenergetic function and enhanced glycolysis dependency in human tumor cells. Am J Physiol Cell Physiol 2007, 292:C125-C136

27. Deberardinis RJ, Sayed N, Ditsworth D, Thompson CB: Brick by brick: metabolism and tumor cell growth. Curr Opin Genet Dev 2008, 18:54-61

28. Lin RJ, Yu HP, Chang BL, Tang WC, Liao CL, Lin YL: Distinct antiviral roles for human $2^{\prime}, 5^{\prime}$-oligoadenylate synthetase family members against dengue virus infection. J Immunol 2009, 183:8035-8043

29. Chang YC, Chiu YF, Ho LL, Ting CT, Shih KC, Curb JD, Chen YD, Li HY, Chuang LM: TCF7L2 genetic variants and progression to diabetes in the Chinese population: pleiotropic effects on insulin secretion and insulin resistance. J Mol Med (Berl) 2010, 88:183-192

30. Livak KJ, Schmittgen TD: Analysis of relative gene expression data using real-time quantitative PCR and the 2(-Delta Delta $\mathrm{C}(\mathrm{T})$ ) method. Methods 2001, 25:402-408

31. Ahamed M, Akhtar MJ, Siddiqui MA, Ahmad J, Musarrat J, Al-Khedhairy AA, AISalhi MS, Alrokayan SA: Oxidative stress mediated apoptosis induced by nickel ferrite nanoparticles in cultured A549 cells. Toxicology 2011, 283:101-108

32. Fritz G, Just I, Kaina B: Rho GTPases are over-expressed in human tumors. Int J Cancer 1999, 81:682-687

33. Mason EF, Rathmell JC: Cell metabolism: an essential link between cell growth and apoptosis. Biochim Biophys Acta 2011, 1813:645654
34. Favre C, Zhdanov A, Leahy M, Papkovsky D, O'Connor R: Mitochondrial pyrimidine nucleotide carrier (PNC1) regulates mitochondrial biogenesis and the invasive phenotype of cancer cells. Oncogene 2010, 29:3964-3976

35. Santamaria G, Martínez-Diez M, Fabregat I, Cuezva JM: Efficient execution of cell death in non-glycolytic cells requires the generation of ROS controlled by the activity of mitochondrial H+-ATP synthase. Carcinogenesis 2006, 27:925-935

36. Mebratu Y, Tesfaigzi Y: How ERK1/2 activation controls cell proliferation and cell death: is subcellular localization the answer? Cell Cycle 2009, 8:1168-1175

37. Lee YJ, Cho HN, Soh JW, Jhon GJ, Cho CK, Chung HY, Bae S, Lee SJ, Lee YS: Oxidative stress-induced apoptosis is mediated by ERK1/2 phosphorylation. Exp Cell Res 2003, 291:251-266

38. Kim R, Tanabe K, Emi M, Uchida Y, Inoue H, Toge T: Inducing cancer cell death by targeting transcription factors. Anticancer Drugs 2003, 14:3-11

39. Kaminishi M: Diversity of gastric carcinogenesis. Oncology 2005, 69 Suppl 1:1-8

40. Wu L, Yan C, Czader M, Foreman O, Blum JS, Kapur R, Du H: Inhibition of peroxisome proliferator-activated receptor-gamma in myeloid lineage cells induces systemic inflammation, immunosuppression and tumorigenesis. Blood 2012, 119:115-126

41. Maniati E, Bossard M, Cook N, Candido JB, Emami-Shahri N, Nedospasov SA, Balkwill FR, Tuveson DA, Hagemann T: Crosstalk between the canonical NF-kappaB and Notch signaling pathways inhibits Ppargamma expression and promotes pancreatic cancer progression in mice. J Clin Invest 2011, 121:4685-4699

42. Quidville V, Segond N, Pidoux E, Cohen R, Jullienne A, Lausson S: Tumor growth inhibition by indomethacin in a mouse model of human medullary thyroid cancer: implication of cyclooxygenases and 15hydroxyprostaglandin dehydrogenase. Endocrinology 2004, 145: 2561-2571

43. Hull MA, Gardner SH, Hawcroft G: Activity of the non-steroidal antiinflammatory drug indomethacin against colorectal cancer. Cancer Treat Rev 2003, 29:309-320

44. Inada T, Kikuyama S, Ichikawa A, Igarashi S, Ogata Y: Bcl-2 expression as a prognostic factor of survival of gastric carcinoma. Anticancer Res 1998, 18:2003-2010

45. Koshida Y, Saegusa M, Okayasu I: Apoptosis, cell proliferation and expression of Bcl-2 and Bax in gastric carcinomas: immunohistochemical and clinicopathological study. Br J Cancer 1997, 75:367373

46. Saegusa M, Takano Y, Okayasu I: Bcl-2 expression and its association with cell kinetics in human gastric carcinomas and intestinal metaplasia. J Cancer Res Clin Oncol 1995, 121:357-363

47. Zafirellis K, Karameris A, Milingos N, Androulakis G: Molecular markers in gastric cancer: can p53 and bcl-2 protein expressions be used as prognostic factors? Anticancer Res 2005, 25:3629-3636

48. Lee HK, Lee HS, Yang HK, Kim WH, Lee KU, Choe KJ, Kim JP: Prognostic significance of $\mathrm{Bcl}-2$ and $\mathrm{p} 53$ expression in gastric cancer. Int J Colorectal Dis 2003, 18:518-525

49. Klein R, Vollmers $\mathrm{H}$, Mullerhermelink $\mathrm{H}$ : Different expression of $\mathrm{Bcl}-2$ in diffuse and intestinal type stomach carcinomas. Oncology Rep 1996, 3:825-828

50. van der Woude CJ, Kleibeuker JH, Tiebosch AT, Homan M, Beuving $A$, Jansen PL, Moshage $\mathrm{H}$ : Diffuse and intestinal type gastric carcinomas differ in their expression of apoptosis related proteins. J Clin Pathol 2003, 56:699-702

51. Jacobson MD, Burne JF, King MP, Miyashita T, Reed JC, Raff MC: $\mathrm{Bcl}-2$ blocks apoptosis in cells lacking mitochondrial DNA. Nature 1993, 361:365-369

52. Dey R, Moraes CT: Lack of oxidative phosphorylation and low mitochondrial membrane potential decrease susceptibility to apoptosis and do not modulate the protective effect of $\mathrm{Bcl}-\mathrm{x}(\mathrm{L})$ in osteosarcoma cells. J Biol Chem 2000, 275:7087-7094

53. Chen ZX, Pervaiz S: Bcl-2 induces pro-oxidant state by engaging mitochondrial respiration in tumor cells [Erratum appeared in Cell Death Differ 2012, 19:551]. Cell Death Differ 2007, 14:1617-1627

54. Chen ZX, Pervaiz S: Involvement of cytochrome c oxidase subunits $\mathrm{Va}$ and $\mathrm{Vb}$ in the regulation of cancer cell metabolism by Bcl-2. Cell Death Differ 2010, 17:408-420

55. Kahl R, Kampkötter A, Wätjen W, Chovolou Y: Antioxidant enzymes and apoptosis. Drug Metab Rev 2004, 36:747-762 
56. Hwang TS, Choi HK, Han HS: Differential expression of manganese superoxide dismutase, copper/zinc superoxide dismutase, and catalase in gastric adenocarcinoma and normal gastric mucosa. Eur J Surg Oncol 2007, 33:474-479

57. Monari M, Foschi J, Calabrese C, Liguori G, Di Febo G, Rizzello F, Gionchetti P, Trinchero A, Serrazanetti GP: Implications of antioxidant enzymes in human gastric neoplasms. Int J Mol Med 2009, 24:693700
58. Monari M, Trinchero A, Calabrese C, Cattani O, Serrazanetti GP, Foschi J, Fabbri A, Zahlane D, Di Febo G, Tonini V, Cervellera M, Tosi MR, Tugnoli V: Superoxide dismutase in gastric adenocarcinoma: is it a clinical biomarker in the development of cancer? Biomarkers 2006, 11:574-584

59. Vanlangenakker N, Vanden Berghe T, Krysko DV, Festjens N, Vandenabeele P: Molecular mechanisms and pathophysiology of necrotic cell death. Curr Mol Med 2008, 8:207-220 